\title{
Record of the Emerald Ash Borer (Agrilus planipennis) in Ukraine is Confirmed
}

\author{
Alexander N. Drogvalenko ${ }^{1}$, Marina J. Orlova-Bienkowskaja ${ }^{2, *}$ and Andrzej O. Bieńkowski ${ }^{2}$ \\ 1 V.N. Karazin Kharkiv National University, 4 Svobody sq., 61022 Kharkiv, Ukraine; triplaxxx@ukr.net \\ 2 A.N. Severtsov Institute of Ecology and Evolution of Russian Academy of Sciences, 119071 Moscow, Russia; \\ bienkowski@yandex.ru \\ * Correspondence: marinaorlben@yandex.ru
}

Received: 13 September 2019; Accepted: 10 October 2019; Published: 11 October 2019

\begin{abstract}
Agrilus planipennis (Coleoptera: Buprestidae) is a devastating invasive pest of ash trees. This wood-boring insect is native to Asia and established in European Russia about 20 years ago. It severely damages Fraxinus pennsylvanica plantations and quickly spreads. In 2019 we first detected A. planipennis in Ukraine. More than 20 larvae were collected from under the bark of $F$. pennsylvanica trees on 5 September 2019 in the Markivka District of the Luhansk Region. The coordinates of the localities of collection were $49.614991 \mathrm{~N}, 39.559743 \mathrm{E} ; 49.614160 \mathrm{~N}, 39.572402 \mathrm{E}$; and $49.597043 \mathrm{~N}, 39.561811 \mathrm{E}$. The photos of the damaged trees with larval galleries, exit holes and larvae are presented. It indicates that A. planipennis is established in the east of Ukraine. This fact is important for development of quarantine protocols to prevent or at least slow the further spread of this invasive pest in Europe.
\end{abstract}

Keywords: emerald ash borer; EAB; Ukraine; Europe; Fraxinus pennsylvanica; ash trees; invasive pest; plant quarantine

\section{Introduction}

The emerald ash borer (EAB), Agrilus planipennis (Coleoptera: Buprestidae), a pest of ash trees (Fraxinus spp.), is native to China, Russian Far East, Japan and Korea [1]. Since its accidental introduction to North America in the 1990s, this devastating pest has widely spread in Canada and the United States and killed hundreds of millions of ash trees [2]. Similarly, the introduction of EAB into Moscow, again back in the 1990s, has likewise led to widespread mortality of $F$. pennsylvanica in an ever-expanding outward range [3,4]. The spread of A. planipennis should be carefully monitored, because it poses a serious threat to F. pennsylvanica plantations in Europe [5]. Before 2019 A. planipennis was recorded only in European Russia. In 2018, it was recorded in the very south of the Voronezh Region of Russia, i.e. near the border of the Luhansk Region of Ukraine [6]. Information about the spread A. planipennis into another country is crucial for plant quarantine protocols. Thus, we decided to look for the pest in the Luhansk Region of Ukraine.

\section{Materials and Methods}

Between 20 and 22 June 2019 ash trees in the Starokozhiv Forest and the field shelter belt in its vicinity (the Markivka District of the Luhansk Region of Ukraine) were examined by A.N. Drogvalenko. This locality was chosen for the survey because it is just about $25 \mathrm{~km}$ from the nearest known A. planipennis locality in Russia [6]. The stems of about 250 ash trees (F. excelsior and F. pennsylvanica) were examined for the characteristic D-shaped exit holes. Three trees of $F$. pennsylvanica damaged by A. planipennis were detected. These trees were situated at the edge of the forest belts and had a diameter of 7-10 cm. Characteristic D-shaped exit holes were situated at a height of $50-200 \mathrm{~cm}$. The infested trees had dying of upper branches, reduced foliage density (small leaves) and fewer seeds. This information was included to the paper posted as a preprint to bioRxiv on 2 July 2019 [7]. 
Immediately following the appearance of this preprint on the Internet, the National Plant Protection Organization of Ukraine conducted an official survey in the same area and did not detect A. planipennis. And since we had no specimens or photos for confirmation, our record of A. planipennis in Ukraine was considered unreliable [8]. A description of the forest provided in the report of the Ukrainian Plant Protection Organization indicates that the employees of this organization did not find the infested forest belt. Unfortunately, they did not ask A.N. Drogvalenko to show them these trees or indicate the exact coordinates of his finding.

Between 4 and 6 September 2019 A.N. Drogvalenko visited the Markivka District of the Luhansk Region of Ukraine again and repeated the survey of the ash trees. His aim was to take photos of the exit holes, larval galleries and larvae of $A$. planipennis, and to collect larvae from under the bark. Larvae were identified by an illustrated guide to distinguish emerald ash borer (A. planipennis) from its congeners in Europe [9].

\section{Results}

The same three infested trees and more than 40 other F. pennsylvanica trees, heavily infested with A. planipennis, were found in this region (Figure 1). More than 20 larvae were collected and preserved in alcohol. The larvae under the bark were found to be of different instars including the last instar. The coordinates of the trees where the larvae were collected are $49.614991 \mathrm{~N}, 39.559743 \mathrm{E} ; 49.614160 \mathrm{~N}$, 39.572402 E; and $49.597043 \mathrm{~N}, 39.561811 \mathrm{E}$ (roadside plantation). The larvae are in the collection by A.N. Drogvalenko in Kharkiv.

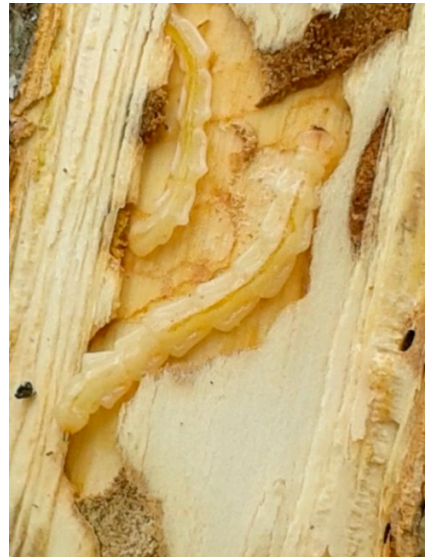

(a)

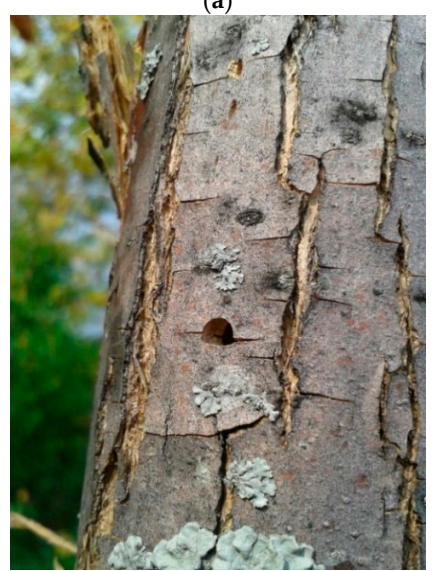

(c)

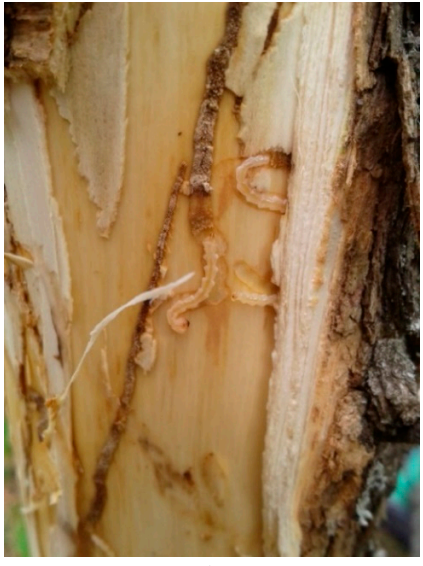

(b)

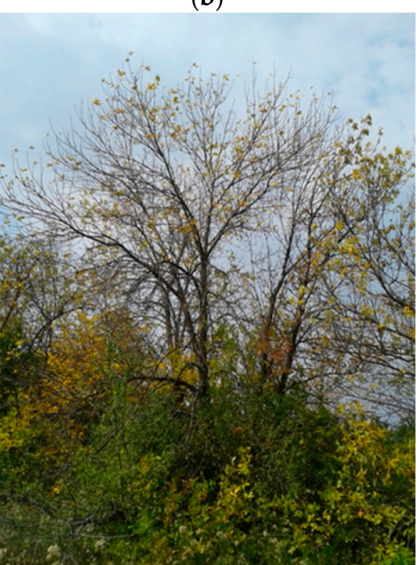

(d)

Figure 1. Symptoms of emerald ash borer (EAB) infestation on ash trees (Fraxinus pennsylvanica) in the Markivka Disrtict of Ukraine. (a,b) Larvae of A. planipennis; (c) exit hole; and (d) a damaged tree.

No infestations of F. excelsior were detected in spite of this ash species being common in this forest. 


\section{Discussion}

Since the examined trees are heavily infested and some larvae are of the last instar, it is obvious that the infestation is at least two years old. The finding of EAB in Ukraine is not surprising. By 2019 EAB has spread to 14 regions of European Russia: Bryansk, Kaluga, Lipetsk, Moscow, Orel, Ryazan, Smolensk, Tambov, Tula, Tver, Vladimir, Volgograd, Voronezh and Yaroslavl [7]. The distance between the entry point of invasion (Moscow) and the most remote known EAB locality (the officially declared plant quarantine zone in Volgograd [10]) is about $900 \mathrm{~km}$. The distance from Moscow to the locality of the EAB detection in Ukraine is about $700 \mathrm{~km}$. Therefore, it is not excluded that EAB is already widespread in Ukraine.

\section{Conclusions}

There is no doubt that A. planipennis is established in Ukraine. It should be taken into account in plant quarantine protocols of European countries.

Author Contributions: Conceptualization, M.J.O.-B.; investigation, A.N.D.; writing-original draft preparation, M.J.O.-B. and A.O.B.; funding acquisition, M.J.O.-B.

Funding: This research was funded by Russian Science Foundation, grant number 16-14-10031.

Conflicts of Interest: The authors declare no conflict of interest. The funders had no role in the design of the study; in the collection, analyses, or interpretation of data; in the writing of the manuscript, or in the decision to publish the results.

\section{References}

1. Orlova-Bienkowskaja, M.J.; Volkovitsh, M.G. Are native ranges of the most destructive invasive pests well known? A case study of the native range of the emerald ash borer, Agrilus planipennis (Coleoptera: Buprestidae). Biol. Invasions 2018, 20, 1275-1286. [CrossRef]

2. Emerald Ash Borer Info. Available online: http://www.emeraldashborer.info/ (accessed on 7 September 2019).

3. Straw, N.A.; Williams, D.T.; Kulinich, O.A.; Gninenko, Y.I. Distribution, impact and rate of spread of emerald ash borer Agrilus planipennis (Coleoptera: Buprestidae) in the Moscow region of Russia. Forestry 2013, 86, 515-522. [CrossRef]

4. Orlova-Bienkowskaja, M.J. Ashes in Europe are in danger: The invasive range of Agrilus planipennis in European Russia is expanding. Biol. Invasions 2014, 16, 1345-1349. [CrossRef]

5. Valenta, V.; Moser, D.; Kapeller, S.; Essl, F. A new forest pest in Europe: A review of Emerald ash borer (Agrilus planipennis) invasion. J. Appl. Entomol. 2017, 141, 507-526. [CrossRef]

6. Baranchikov, Y.N.; Demidko, D.A.; Seraya, L.G. A quarter-century of emerald ash borer in Europe. In Proceedings of the Conference Preparing Europe for Invasion by the Beetles Emerald Ash Borer and Bronze Birch Borer, Two Major Tree-Killing, Vienna, Austria, 1-4 October 2018; Mayer, P., Ed.; BFW: Vienna, Austria, 2018; p. 9.

7. Orlova-Bienkowskaja, M.J.; Drogvalenko, A.N.; Zabaluev, I.A.; Sazhnev, A.S.; Peregudova, E.Y.; Mazurov, S.G.; Komarov, E.V.; Bienkowski, A.O. Bad and good news for ash trees in Europe: Alien pest Agrilus planipennis has spread to the Ukraine and the south of European Russia, but does not kill Fraxinus excelsior in the forests. bioRxiv 2019, 689240. [CrossRef]

8. EPPO Global Database. Agrilus planipennis Does Not Occur in Ukraine. EPPO Reporting Service (2019/156). Available online: https:/gd.eppo.int/reporting/article-6586 (accessed on 7 September 2019).

9. Volkovitsh, M.G.; Orlova-Bienkowskaja, M.J.; Kovalev, A.V.; Bieńkowski, A.O. An illustrated guide to distinguish emerald ash borer (Agrilus planipennis) from its congeners in Europe. Forestry 2019. [CrossRef]

10. Rosselkhoznadzor (2019) Russian Federal Service for Veterinary and Phytosanitary Surveillance. Open Data Russia. Available online: https://data.gov.ru/opendata/7708523530-carantinzone (accessed on 26 June 2019). 